\title{
Axillary skin excision as a treatment for axillary hyperhidrosis
}

\author{
P. C. Weaver \\ M.B., F.R.C.S., F.R.C.S.Ed. \\ Senior Surgical Registrar, Department of Surgery, \\ Westminster Hospital, London
}

\begin{abstract}
Summary
This report describes three severe cases of axillary hyperhidrosis successfully treated with the HurleyShelley operation. It appears to be a simple and effective method of dealing with a condition which is certainly not rare and in its most severe form can be a great social embarrassment to the patient.
\end{abstract}

Axillary skin excision in the management of axillary hyperhidrosis was first described by Hurley $\&$ Shelley in 1963 . They explain that they discovered this treatment accidentally in 1957 during an investition of the histology of axillary sweat glands. A skin biopsy specimen taken from the axilla of a patient with intense hyperhidrosis produced a remarkable reduction in the amount of sweat in this axilla. Using Randall's method (1946) for assessing the distribution of sweat glands in man with starchpaper-iodine, they observed that $70-80 \%$ of axillary sweat is produced by glands found in the dome or central portion of the axilla. Having mapped out this region, an ellipse of this central axillary tissue was excised down to and including part of the subcutaneous tela, thus removing a sufficient number of the most active sweat glands in the axilla. The elliptical excision is placed transversely, i.e. across the mid axilla, and not in a longitudinal axis. After haemostasis, the skin edges are opposed with vertical mattress sutures. Hurley \& Shelley carry out this procedure under local anaesthesia. In their initial paper they describe the effect of this operation on four patients and in their second paper, on twelve patients. In only one case in this series was the result thought to be of little or no effect. Interestingly enough, the value of this procedure does not seem to be widely appreciated in this country and even though the method has been used with some success in Britain, no evaluation of it has yet appeared in British literature. The purpose of this paper is to present three cases treated successfully with the above method and to draw attention to the value of this treatment in suitable cases.

\section{Case reports}

Case 1. A.E.

A male, aged 42, employed in a bank, was referred by his general practitioner for consideration of cervico-thoracic sympathectomy. Axillary hyperhidrosis commenced a year prior to his referral. It soon became extremely tiresome and caused extreme embarrassment. Sweating stained his clothes to such an extent that the dry cleaners were unable to remove these marks and the patient spent more than $£ 100$ on new clothes in less than 6 months. The patient had tried all known anti-perspirants and deodorants with no effect. There was no family history of hyperhidrosis. General examination revealed a fit, healthy man and investigations revealed no evidence of hyperthyroidism. Thoracic inlet and chest X-rays, ECG and urinary steroids were normal.

\section{Treatment}

$10 \%$ iodine in starch powder was applied to both axillae on a small cotton wool pad, after initially washing and drying the axillae with dry gauze. After 2 or $3 \mathrm{~min}$, sweat from the glands in the central portion of the axillae had turned the starch iodine powder dark blue. The stained zone measured $4 \times$ $6 \mathrm{~cm}$. Under general anaesthesia, bilateral elliptical incisions were made in the axillae and placed transversely in the line of the skin crease. They were both $7 \mathrm{~cm}$ long and $3 \mathrm{~cm}$ wide at the centre. The skin edges were undercut by $1 \mathrm{~cm}$ on either side removing subcutaneous glandular tissue. Warm wet swabs were placed in the wounds and pressure applied for $5 \mathrm{~min}$. Any remaining bleeding points were diathermied. After haemostasis the skin edges were apposed with interrupted silk mattress sutures and a light pressure dressing applied for the first $24 \mathrm{hr}$. The patient was then discharged from hospital and returned 8 days later for removal of his sutures (Fig. 1.). Wound healing was satisfactory and the operation caused minimal discomfort. The patient was delighted with the result and is symptom-free after a period of 9 months.

\section{Case 2. C.D.}

Male, aged 20, a university student, was referred from the Department of Dermatology at the Westminster Hospital following the demonstrated success of our first patient. This patient was an anxious, rather highly strung young man with generalized hyperhidrosis which was much worse in his axillae 


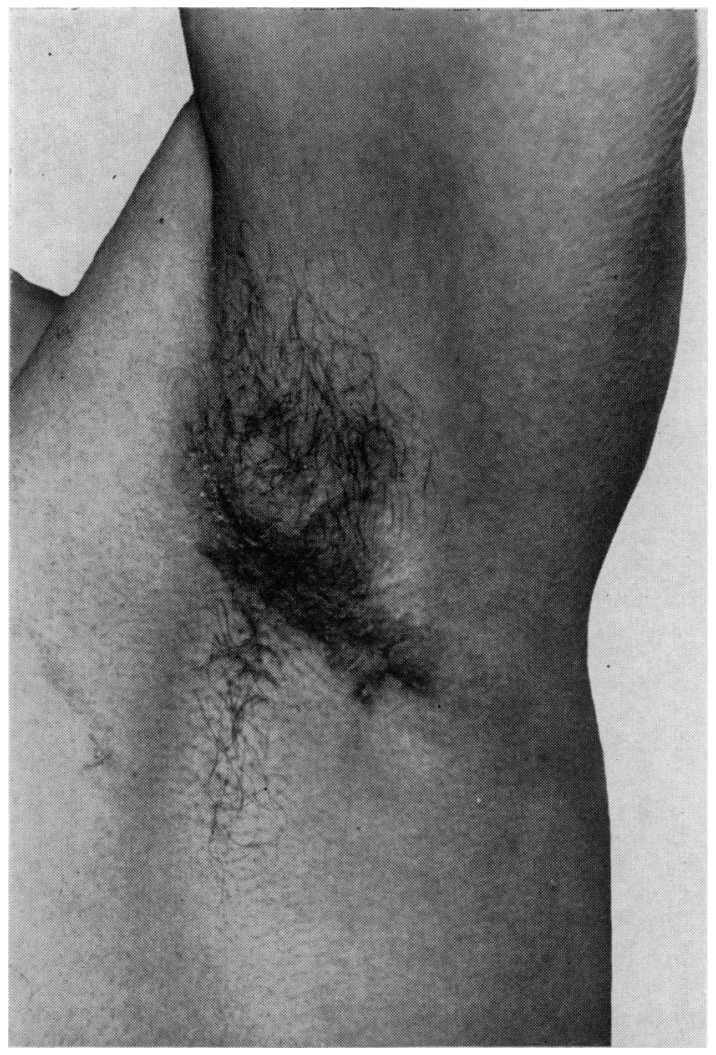

FIG. 1 (Case 1). Appearance of the left axilla after a period of 3 weeks.

than other regions of his body. Again, this patient was disturbed by sweat-staining of his clothes under the axillae. He was treated in the same way as the previous patient, though starch iodine marking of the main sweating areas in his axillae showed these areas to be more diffuse but with an area of central concentration of sweating at the apex of the axillae of $4 \times 4 \mathrm{~cm}$. Surgical treatment under general anaesthesia was carried out as before (Fig. 2). Again, the axillary skin healed satisfactorily and on follow-up 6 months later the patient was much more comfortable than previously and now had no excessive sweating in the axillae.

\section{Case 3. L.H.}

Female, aged 17, a receptionist, engaged to be married within a few months, was referred by her general practitioner with severe axillary hyperhidrosis which had commenced a year prior to her referral. Again, anti-perspirants had proved ineffective. Sweat-staining of her clothes had become a considerable embarrassment. Family history was negative. Chest X-ray, thyroid function tests, and urinary steroids were normal. Fig. 3 shows both her

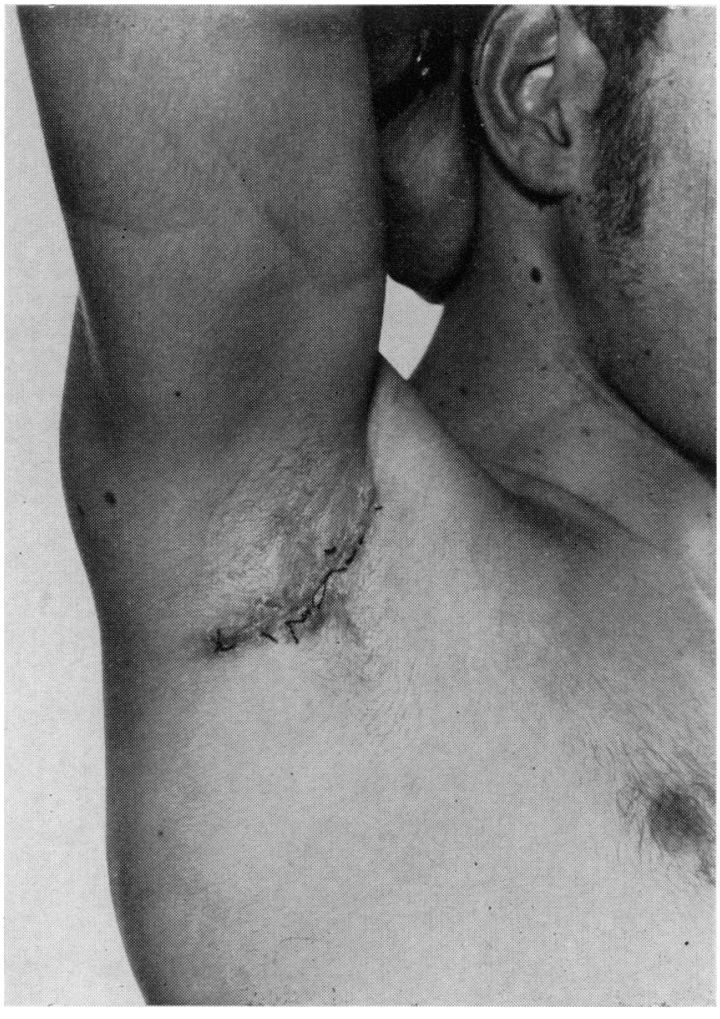

Fig. 2 (Case 2). One of the incisions 8 days after operation and prior to the removal of the sutures.

axillae stained with starch iodine and these areas separated by lines drawn by a marker pen. Surgical treatment was carried out as before under general anaesthetic and the patient discharged from hospital after $24 \mathrm{hr}$. Sutures were removed 8 days later. The patient was again delighted by the result and has remained symptom free to date (12.5.70).

\section{Discussion}

Topical and oral anticholinergic drugs are used in the treatment of axillary hyperhidrosis (Brown \& Sandler, 1951; Zupko, 1954; Orentreich \& Rein, 1957; Knudsen \& Meier, 1963). Most dermatologists however, find their use uniformly disappointing. More recently topical scopolamine esters have been used to suppress axillary sweating (MacMillan, Reller \& Snyder, 1964; Stoughton et al., 1964). Surgical management of axillary hyperhidrosis includes cervico-thoracic sympathectomy, with the removal of the 4th and 5th thoracic ganglia (MacGregor, 1955; DeTakats, 1957) and a local surgical technique involving dissection of the glandular layer of the axilla, described by Skoog \& Thyresson, 1962. Apart from the greater surgical hazards associated with sympathectomy over this simple pro- 


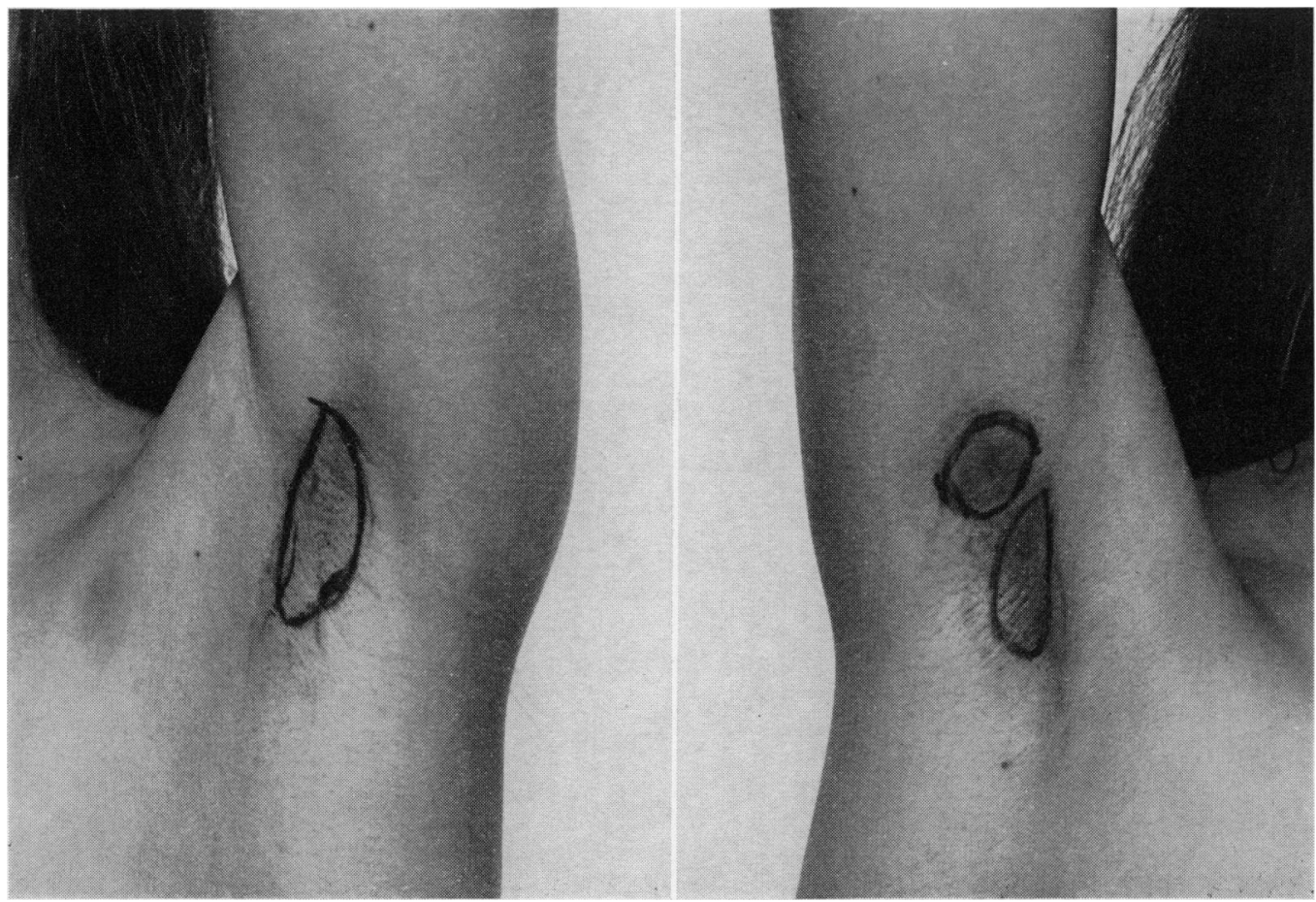

cedure, is the occasional disadvantage of the compensatory thermal hyperhidrosis, principally of the trunk, that may ensue (Shelley \& Florence, 1960). The Skoog-Thyresson procedure is essentially a broad dissection of virtually all the axillary sweat glands and no attempt is made to identify the foci of high sweat delivery areas in the axillae.

The advantages of the Hurley-Shelley operation are:

1. That it attempts to deal with the main sweatbearing area.

2. That it does not affect sweating in other parts of the body.

3. That it is a very simple operation which can be carried out under local anaesthesia, if desired.

4. That there is little chance of damage to major axillary vessels or lymphatics, if reasonable care is taken.

\section{Acknowledgments}

The author wishes to thank Mr Charles Drew and Dr Peter Copeman for their help and encouragement, and permission to describe these cases.

\section{References}

BRown, C.S. \& SANDLER, I.L. (1951) Effective application of banthine in dermatology. Archives of Dermatology and Syphilis, 64, 431

DeTakats, G. (1957) Surgical treatment of hyperhidrosis; types of sweating, tests of sweating, surgical treatment, results. Archives of Dermatology, 76, 31
Hurley, H.J. \& Shelley, W.B. (1963) A simple surgical approach to the management of axillary hyperhidrosis. Journal of the American Medical Association, 186, 109.

Hurley, H.J. \& Shelley, W.B. (1966) Axillary hyperhidrosis; clinical features and local surgical management. British Journal of Dermatology, 78, 127.

KnUdSEN, E.A. \& MeieR, C.H. (1963) Treatment of hyperhidrosis with topical propantheline bromide. Acta Dermatovener (Stockholm), 43, 154.

MACGREGOR, A.L. (1955) Surgery of the Sympathetic. John Wright, Bristol.

MacMillan, F.S., Reller, H.H. \& SNyder, F.H. (1964) The antiperspirant action of topically applied anticholinergics. Journal of Investigative Dermatology, 43, 363.

Orentreich, N. \& ReIN, C.R. (1957) The effect of diphemanil methylsulfate on hyperhidrosis. Archives of Dermatology, 76, 762.

RANDALL, W.C. (1946) Quantitation and regional distribution of sweat glands in man. Journal of Clinical Investigation, 25, 761 .

Shelley, W.B. \& FloRence, R. (1960) Compensatory hyperhidrosis after sympathectomy. New England Journal of Medicine, 263, 1056.

Skoog, T. \& Thyresson, N. (1962) Hyperhidrosis of axillae, method of surgical treatment. Acta Chirurgie Scandinavica $124,531$.

Stoughton, R.B., Chiu, F., Fritsch, W. \& Nurse, D. (1964) Topical suppression of eccrine sweat delivery with a new anticholinergic agent. Journal of Investigative Dermatology 42, 151.

ZUPKo, A.G. (1954) Evaluation of prantal cream in localized hyperhidrosis. American Journal of Pharmacology, 126, 194. 